\title{
Erythroid Stem Cell
}

National Cancer Institute

\section{Source}

National Cancer Institute. Erythroid Stem Cell. NCI Thesaurus. Code C12526.

A unipotent hematopoietic progenitor cell derived from myeloid stem cells that is committed to the erythrocyte cell lineage. 